Published in final edited form as:

J Adv Nurs. 2020 February ; 76(2): 706-714. doi:10.1111/jan.14007.

\title{
Strategies for overcoming language barriers in research
}

\author{
Allison Squires, PhD, RN, FAAN ${ }^{1,2}$ [Associate Professor, Research Assistant Professor], \\ Tina Sadarangani, PhD, APRN-BC ${ }^{1}$ [Assistant Professor], Simon Jones, PhD $^{3}$ [Professor] \\ ${ }^{1}$ Rory Meyers College of Nursing, New York University, New York City, New York \\ ${ }^{2}$ School of Medicine, New York University, New York City, New York \\ ${ }^{3}$ Population Health, School of Medicine, New York University, New York City, New York
}

\begin{abstract}
Aim-This paper seeks to describe best practices for conducting cross-language research with individuals who have a language barrier.

Design—Discussion paper.

Data Sources-Research methods papers addressing cross-language research issues published between 2000-2017.

Implications for Nursing-Rigorous cross-language research involves the appropriate use of interpreters during the research process, systematic planning for how to address the language barrier between participant and researcher and the use of reliably and validly translated survey instruments (when applicable). Biases rooted in those who enter data into "big data" systems may influence data quality and analytic approaches in large observational studies focused on linking patient language preference to health outcomes.
\end{abstract}

Conclusion-Cross-language research methods can help ensure that those individuals with language barriers have their voices contributing to the evidence informing healthcare practice and policies that shape health services implementation and financing. Understanding the inherent conscious and unconscious biases of those conducting research with this population and how this may emerge in research studies is also an important part of producing rigorous, reliable, and valid cross-language research.

- $\quad$ This study synthesized methodological recommendations for cross-language research studies with the goal to improve the quality of future research and expand the evidence-base for clinical practice.

\footnotetext{
Correspondence: Allison Squires, Rory Meyers College of Nursing, New York University, 433 First Avenue, 6th Floor, New York, NY 10010. aps6@nyu.edu.

7.AUTHOR CONTRIBUTIONS

AS, TS and SJ: Made substantial contributions to conception and design, or acquisition of data, or analysis and interpretation of data. AS, TS and SJ: Involved in drafting the manuscript or revising it critically for important intellectual content. AS, TS and SJ: Given final approval of the version to be published. Each author should have participated sufficiently in the work to take public responsibility for appropriate portions of the content. AS, TS and SJ: Agreed to be accountable for all aspects of the work in ensuring that questions related to the accuracy or integrity of any part of the work are appropriately investigated and resolved.

6. CONFLICT OF INTEREST

The authors declare that they have no conflicts of interest.
} 
- Clear methodological recommendations were generated that can improve research rigor and quality of cross-language qualitative and quantitative studies.

- The recommendations generated here have the potential to have an impact on the health and well-being of migrants around the world.

\section{Keywords}

asylum seeker; immigrants; language barrier; methods; nurses; nursing; refugee; translations; undocumented immigrants

\section{INTRODUCTION}

Global migration has reached unprecedented levels in human history in the twenty-first century, with $3.3 \%$ of the world's population having migrated internationally and 740 million people have migrated in their own countries (International Organization for Migration, 2017). Medical tourism is also on the rise and language concordance is not always a guarantee between nurses and their patients in those cases (Kanchanachitra et al., 2011; Reitig \& Squires, 2015). For nurses and other healthcare researchers, migration creates a common challenge for healthcare research: language barriers.

Individuals with language barriers present new opportunities and challenges for researchers seeking to strengthen the evidence-base for clinical nursing practice and education around the world. Research with this population is also critical for understanding health outcomes, how individuals who have moved countries access (or the barriers to) health services and developing and testing effective strategies for health literacy promotion to name a few. Research will also help ensure that individuals with language barriers do not face discrimination in their health systems and subsequently develop costly health disparities.

It is surprising, however, how many researchers do not minimize the threats to research rigor posed by language barriers with their subjects. Patient and health services focused research on language barriers has historically been lacking and limited to a small group of researchers globally, even as incentives for such research have appeared in many countries (Schwei et al., 2016). In the case of nursing, the literature has focused more on researching the language skills (or lack thereof) of internationally educated nurses rather than their patients (Allan \& Westwood, 2016; Müller, 2016). A cursory search of PubMed and CINAHL reveal that publications linked to nurses about language barriers since the year 2000 number, after removal of duplicates, only 280 of 303. Approximately one third of those are practice-based papers not involving research, editorials, or opinions-the lowest level of the evidence-based practice pyramid. Whilst there may be more publications, inappropriate use of key words may keep many hidden from systematic searches.

In this discussion paper, we draw from an international, interdisciplinary body of research that has explored and successfully addressed methodological challenges of qualitative and quantitative research involving language barriers in health care, known as cross-language research methods. We seek to highlight the key methodological implications of doing research involving language barriers by drawing from methodological developments in the 
literature since the year 2000 through 20,217-a period representing unprecedented growth in scientific studies and cross-language methodological developments. Both qualitative and quantitative methodological implications are reviewed from selected studies.

\section{BACKGROUND}

This section provides an overview of two key areas associated with cross-language research in nursing: language barriers and interpreter types. It aims to provide the reader with a basic conceptual understanding of core linguistic principles involved with addressing language barriers in research.

\subsection{Language barriers in nursing and health care}

Language barriers have been part of nursing practice since the formal inception of the profession in the nineteenth century when Nightingale was caring for soldiers from across Europe during the Crimean War. In the twenty-first century with global migration rates at record levels, language barriers present multiple challenges for health systems delivery (Bloemraad \& Sheares, 2017; Czaika \& de Haas, 2013).

Research is needed to devise the best, context specific strategies for meeting the needs of patients with language barriers. Conducting research in a patient's preferred language offers the best opportunity to truly capture reliable and valid results representative of their experiences. A preferred language is the person's "language of the heart", the one that they want to speak when they feel at their most vulnerable. The conduct of research when a language barrier is involved has two aspects. First, understanding linguistic competency and literacy of participants and planning the study around those factors. The second aspect focuses on addressing the language of health care and research itself, known as language for specific purposes (LSP).

First, to be able to communicate effectively with another person individuals need to have what linguists call discourse competence in a language. That means they can have a conversation with someone relatively easily and do not have to stop and look up words or phrases (Danesi, 1996; Savignon, 1997; Squires, 2008). For example, immigrant children may have this level of language competence, but they usually lack a more sophisticated understanding of the language since most of the time, they do not receive formal education in their parents' language. They will not have the vocabulary to speak "health care" either. They are known as "heritage" speakers of a language (Montrul, 2010).

Health care, as we know, is its own language and fits the criteria of a language for a specific purpose (Hull, 2016). LSP is the vernacular of the discipline. The professions all have their own language as do the different realms of the social sciences. When students study to become members of the discipline or profession, part of their socialization is learning the language. Therefore, effective communication with LSP depends on the person's ability to translate not only the disciplinary vernacular, but also the standard language (Hull, 2016).

We see "failures" of translating LSP with patients who speak our own language when we cannot improve their health literacy. In the case of language translation issues between 
patients and providers, for example, miscommunication related to translation increases the patient's risk for hospital readmission, adverse events, and delays in care, to name a few (De Gagne, Oh, So, \& Kim, 2014; Dowsey, Broadhead, Stoney, \& Choong, 2009; Durstenfeld, Ogedegbe, Katz, Park, \& Blecker, 2016; Karliner, Kim, Meltzer, \& Auerbach, 2010; Whittal \& Lippke, 2016). Therefore, the same threats mistranslation poses to patients' health and well-being will threaten the rigor of research studies involving translation.

\subsection{An overview of types of healthcare interpreters and their potential research roles}

Interpreters are an important part of mitigating threats to rigor in research. There is a difference, however, between an interpreter and a translator. An Interpreter is a person who conducts "live" interpretation between two people. A Translator is someone who translates text-based documents between the source language and the target language (Squires, 2008; Temple, 2002). Qualified interpreters and translators will have had their language skills formally evaluated by an independent source (Hull, 2016). The next sections focus on the roles of interpreters in research with roles of translators discussed specifically in the section focused on research implementation.

To begin, in the case of interpreters, they will play an important role in research data collection and potentially, analysis. Any interpreter contributing to a study ideally will have some experience facilitating research implementation, but that is not always possible (Squires, 2008). There are five types of interpreters or services that can be used in research, each with pros and cons and budgetary implications.

The first type of interpreter we will discuss is the Dual Role Interpreter. This is usually a healthcare provider who has had their language proficiency formally evaluated by an independent source. They may have grown up speaking the language and continued studying it as they progressed in their education or alternatively, learned the language through intensive study or living and working abroad. In many countries, it is common for healthcare providers to speak multiple languages, especially when there are several official languages in a country. Again, it is important to remember that their level of language proficiency for healthcare language may vary.

The advantage of the dual role interpreter (especially if they also have research training) is that their contributions to the study will be informed by their experiences working with patients with language barriers. There is the potential for a more nuanced understanding of experiences or the challenges of measurement with specific populations with language barriers. A dual role interpreter, however, may bring their own set of biases into how results are interpreted from the study because of their experiences. This aspect of interpreter identity, even in quantitative studies, should be factored into study design and discussed in the limitations.

An in-person interpreter is an individual who has received specialized training. In health care, they have learned healthcare vocabulary as part of their training so they can effectively translate health care's language - a definite advantage for study implementation. For research purposes, these interpreters are the best option if the study will involve 
communicating complex healthcare information to a study participant, such as might occur in randomized controlled trials.

In-person interpreters in research can also have no healthcare experience, which can provide an advantage for researchers who seek to minimize bias in participant responses in studies involving the patient experience. Like any healthcare worker, healthcare interpreters may have their own biases from interactions with healthcare providers, navigating systems issues and memorable patient interactions. Even though they may try to stay objective, the risk for them inserting bias into the findings increases if this threat to rigor is not managed well.

Technology-based interpreting is the kind that most clinicians are familiar with and it comes in the form of telephone or video-based interpreting services. When research funds are limited, using this service —especially if it is already part of the institution's resources—can offer the most cost-effective option for conducting research. A threat to rigor from this type of interpreting is that the technology- based interpreting industry is largely unregulated globally and relies on companies to conduct their own internal quality checks of interpreter performance.

Finally, two other ways of bridging language barriers could threaten the rigor of research findings: Online translation services and family members. Online translation services have not yet developed the ability to effectively translate health care's language. While they can seem like a good way to translate, for example, transcripts in qualitative research, many translation errors will happen-especially with patient interviews because they may often use obscure slang words from their particular dialect that the computer will mistranslate. A qualified translator will still be needed to check the translation, which could still take just as much time as if they did it themselves.

Family members may or may not make for effective interpreters for a research study. Just like during a healthcare encounter where sensitive or culturally taboo topics may emerge; the family member may influence the translation and information provided. Unless the study design includes the family member in it, using independent interpreters presents the best mediating option for threats to rigor from translation by family members. Nonetheless, with the participant's consent, a family member could help enhance data quality by either improving its precision (in the case of a survey) or helping the participant to remember important experiential details (in the case of qualitative research).

\section{DATA SOURCES}

Cross-language research refers to research studies where a language barrier is present and data collection must involve the use of interpreters at some stage during the research process (Croot et al., 2011; Squires, 2009; Squires et al., 2013). A critical factor of crosslanguage research, regardless of methodological approach, is that it must be completed in teams (Chapple \& Ziebland, 2018; Esposito, 2001; Im et al., 2017; Paulus, Jackson, \& Davidson, 2017; Shordike et al., 2010; Stanley \& Slattery, 2003). Cross-language research cannot be rigorous unless a team was involved because the interpretation of the data would be subject to the individual biases of a single researcher and are likely to be less representative of the 
population of interest. The team will include the researcher, coinvestigators, project managers and very importantly, interpreters. The following recommendations were drawn from 73 methods articles addressing some dimension of cross-language qualitative or quantitative research published between 2000 and 2017.

\section{IMPLICATIONS FOR RESEARCH DESIGN}

When the target population for a research study has a language barrier, careful planning is required. In this section, we offer considerations for the design of qualitative and quantitative studies where language barriers are an issue that could threaten the rigor of a study.

\subsection{Qualitative research considerations}

Cross-language qualitative research has grown extensively since the year 2000. Methods have evolved and several common methodological considerations emerged. Importantly, cross-language researchers uniformly agree that translation poses a threat to the trustworthiness of qualitative data (Court \& Abbas, 2013; Esposito, 2001; Im et al., 2017; Jones \& Boyle, 2011; Larkin, Dierckx de Casterlé, \& Schotsmans, 2007; MacKenzie, 2015; Temple, 2002, 2005; Temple \& Young, 2004; Wong \& Poon, 2010; Xian, 2008). Squires (2009) developed criteria from a systematic review of cross-language studies for evaluating how researchers managed translation and then Croot et al. (2011) tested the criteria. The latter concluded that the criteria offered researchers useful direction both with study design and critical appraisal of existing studies, albeit with several caveats related to resources dictating interpreter usage.

Another point of consensus in cross-language research is that interpreter identity matters, with pros and cons for each choice made. Interpreters with translation work experience are uniformly recommended for cross-language studies to minimize the threats to trustworthiness of results posed by translation. The use of students, undergraduate or graduate, for interpreting may create good research socialization opportunities, but could also affect data quality due to their inexperience with both research and translation (Lincoln, González y González, \& Aroztegui Massera, 2016).

Interpreter timing during data collection also matters and well-planned studies account for this factor during study design (Im et al., 2016; Santos, Black, \& Sandelowski, 2014). Timing is rooted in the role design of the interpreter in the study. Researchers may find Role Theory useful in interpreter role design when planning a study (Lynch, 2007; Morgeson, Delaney-Klinger, \& Hemingway, 2005). For example, a functionalist role for an interpreter means the expectation of the interpreter is to adhere to their essential function: interpretation and translation. This would be defined as the "correct behaviours" that functionalist role theory emphasizes. Timing would be limited to the interview data collection point, transcription (if that is part of their role) and translation of the transcript.

An interpreter in an interactionist role, however, offers flexible boundaries to the interpreter that are less proscribed (Lynch, 2007). Interactionist contributions of the interpreter would include not only interpretation and translation of the data, but also contributions to data analysis (Squires, 2008). An interactionist role also allows the interpreter to integrate their 
role as a cultural broker between the parties, thereby contributing potential explanations to themes and categories that have emerged in the analysis or by providing culturally appropriate names for them.

Finally, transcription quality is always a critical part of any qualitative research study and this is where translators will help mitigate threats to rigor (Poland, 1995; Tilley, 2003). Transcriptions are the final point of interpreter-mediated vulnerability in a research study because the quality of translation will affect the entire data analysis process. Clark, Birkhead, Fernandez, and Egger (2017) offer useful recommendations for quality checking the transcription and translation process. These include two independent checks on translation, hiring professional transcription services, achieving consensus around the translation of culturally unique words and slang phrases with the minimal goal of achieving semantic equivalence and the aspirational goal of conceptual equivalence.

\subsection{Quantitative research considerations}

Language barriers or patient language preferences can affect any kind of quantitative study. Most observational studies (e.g., cross-sectional, cohort, case control, etc.) and randomized control trials may involve the use of some survey design and methodological consensus has emerged around what constitutes rigorous survey instrument translation. "Big Data", being newer, means that we do not fully understand where language preference and language barriers manifest themselves in patient outcomes in large datasets. Nonetheless, there are still salient points for discussion even in the early stages of the science.

4.2.1. Survey instruments and translation-There are many instruments with existing reliable and valid translations, or so it may appear. When making the method choice to use an existing translation, researchers should first research the history of the instrument to determine: (a) when it was developed; (b) how it was psychometrically evaluated in its original language; and (d) when and how the translation was completed. Flaws in the process tied with the original instrument translation process will not produce reliable and valid results in the translation. There is also the possibility that factor structures and other psychometric measures may change across cultures and contexts (Brzyski, Kózka, Squires, \& Brzostek, 2016; Choi et al., 2009; Mallinckrodt \& Wang, 2004; Yu, Lee, \& Woo, 2004). Sometimes these changes are not significant, but the researcher must differentiate when they are or are not using the appropriate methods. Just because it has been published does not mean it is a good quality translation. A translator is critical to help evaluate the quality of the survey's translation during this phase of study planning. Some surveys, like the Maslach Burnout Inventory, have professional translations that are available, protected by copyright and may require a fee for their use in research studies (Squires et al., 2014). Failure to appropriately use this kind of survey translation may place the researcher at risk for copyright violations.

Survey instrument translation appropriateness with specific populations is also affected by nativity and dialects - both of which are associated with social risk factors influencing health outcomes (National Academies of Sciences Engineering \& Medicine, 2017). Many countries have multiple official languages where citizens will speak all of them or at least 
one or two with a high level of proficiency. They may not read or write in the other languages.

Examples of this phenomenon come from every part of the world. In Sub-Saharan Africa, it is common for people to speak their tribal language (which may or may not be written), the language of their former colonizers and other languages common to the economic engine of the country (Levin, 2006). Latin America has 448 indigenous languages spoken there, aside from Spanish, Portuguese, or French. China has the unifying scholarly language of Mandarin, with Cantonese the second most spoken language in the country. Yet each village and region in China can have a sub-dialect that only people from those regions understand (Aroian, Wu, \& Tran, 2005; Chidarikire, Cross, Skinner, \& Cleary, 2018). Former Soviet Union States may still speak Russian but most have reasserted their country's language as the primary language (Shpilko, 2006). The languages of India and Pakistan remain numerous as well (Abdelrahim et al., 2017). In the Middle East, Arabic is much like Spanish where the version of the language is specific to the country of origin of the person speaking it. Academic Arabic could be read by any educated person, but dialect specificity is important for accurate translation (Al-Amer, Ramjan, Glew, Darwish, \& Salamonson, 2016). For survey research, this means that a translation of a survey may not work well if it does not match the participant's nativity—especially when measuring symptoms, coping strategies and other health related phenomenon where slang and linguistic variation by country become measurement factors.

Nonetheless, consensus has emerged in several areas around appropriate strategies for ensuring the most reliable, valid and culturally appropriate translation of a survey instrument. Flaherty et al. (1988) were one of the first groups to set early criteria for evaluating instrument translations. Their criteria include researchers taking steps during the translation process to ensure conceptual, semantic, technical, content, and construct equivalence. In time, it has also become clear that forward and backward translation alone are insufficient to ensure reliable and valid translations because that process alone cannot meet the five measures of equivalence (Maneesriwongul \& Dixon, 2004; Perneger, Leplège, $\&$ Etter, 1999; Squires et al., 2013). Systematic approaches to survey instrument translation, therefore, offer the best option to ensure reliable, valid, and culturally representative translations. Most of these approaches offer some combination of content validity indexing (Brzostek et al., 2015; Liu, Squires, \& You, 2011; Squires et al., 2012, 2013, 2014), cognitive interviewing (Benitez \& Padilla, 2014; Benitez, Padilla, van de Vijver, \& Cuevas, 2018; Park, Sha, \& Willis, 2016; Reeve et al., 2011), and interpreter timing (Cha, Kim, \& Erlen, 2007; Erkut, 2010; Johnson, 2006; Sidani, Guruge, Miranda, Ford-Gilboe, \& Varcoe, 2010; Weeks, Swerissen, \& Belfrage, 2007; Xian, 2008; Yu et al., 2004). Finally, conducting pre-data collection evaluations of the survey instrument with the migrant population will not only help determine if they understand the questions being asked in the instrument, but if the wording and literacy level is appropriate for the local population being studied.

4.2.2. Big data, patient language preference, and large dataset observational studies-Whilst there is a huge attraction to use big data to analyse health related issues tied to patient language preference, bigger does not always mean better (Cohen et al., 2015; O'Halloran, Tan, Pham, Bateman, \& Vande Moere, 2018). In addition to the many known 
problems that come with working with large datasets in health care, isolating language barrier related relationships and effects have their own challenges in observational studies. First, data generated from administrative files is not collected with the rigor and consistency that aligns with research practices. It requires multiple people and multiple incentives to get it right. Consequently, organizations that do not place a value on capturing patient language preference will likely have poor data to work with as capturing patient language preference data reflects organizational values for caring for migrants.

A logical leap from this issue then would be the use of missing data management techniques. Patient language preference data are often missing, thus presenting unique challenges for data analysis that are often reflective of biases and potentially, prejudices of who entered the data. Thus, missing language preference data are unlikely to be missing at random. For example, research from the UK shows that it is common to have missing race/ ethnicity classifications of "white" (Tippu et al., 2017). That missing data, with no language preference recorded, would leave out whites who do not speak English yet still comprise a substantial portion of the migrant population in the country. Other datasets might reflect similar patterns.

Another issue affecting large datasets where researchers want to consider language preference is that there are no standards for recording it in health care in terms of naming languages in EHRs. For example, organizations may have a language preference option of "Chinese", when in fact there is no such language. Mandarin is the official language of China and Cantonese is a widely spoken dialect in the country that uses the same alphabet. As previously mentioned, China has multiple dialects—some just at the village level —and those are rarely considered for language preference. For older patients who may migrated in the twentieth century, their village dialect may represent the language of their heart.

Lastly, when trying to use large datasets to compare the impact of language preference on patient outcomes, how demographics are measured and have changed over time become important methodological considerations. In societies that were largely linguistically homogenous before the population changed, these differences present unique challenges for healthcare research. Longitudinal studies, therefore, become critical for studying the impact of changes in language preferences of populations over time and how these impact health outcomes and any resulting disparities.

\subsection{Budgeting for interpretation and translation}

Interpreting and translation can add significant costs to a research study. Costs are determined based on (a) the language being translated; (b) the source and target languages for translation; and (c) the extent of interpreter involvement in the research process.

Straightforward translation of survey instruments or transcription and translation of qualitative interviews have costs dictated by the time involved and the country where the researchers are seeking services. Many professional translation companies contract with translators in other countries to ensure the best quality translation and in some cases, this can save on costs. Country specific translations also ensure that the slang words or other country 
specific vernacular receives the correct interpretation. Professional translation services, however, are the most expensive.

The alternative is to hire research team members who speak the same language as the target population of the study. This can involve hiring research assistants on a part- or full-time basis and the total costs of their participation will depend on the country's labour laws or organization's employment requirements. Funding for the study may limit how long interpreters can be involved with it unless they can contribute to other parts of the study besides data collection. If researchers do want their language concordant staff to remain part of the entire study, they should budget funds to support their involvement for the duration. Depending on the funding source, this may or may not contribute significantly to the study's total costs.

\section{DISCUSSION AND CONCLUSION}

Research will play a critical role in helping health systems, workers and patients determine the most effective ways to bridge language barriers between patients, providers and systems in ways that optimize health and system outcomes. Improved research rigor in studies involving language barriers in health care are also needed to create evidence-based policies at the organizational, local, national, and international levels.

All recommendations are made mindful of the possibility that any researcher or member of their team could be subject to ethnocentric assumptions in their work and thus, should operate from a place of being conscious of their own biases when implementing and interpreting studies involving language barriers. Using cross-language research methods to generate better, more rigorous evidence specific to the experiences of people with language barriers is critical for strengthening health care's evidence base that informs clinical practice and policy.

\section{Acknowledgments}

Funding information This paper was informed by research funded by the United States' Agency for Health Care Research and Quality, R01HS23593.

\section{REFERENCES}

Abdelrahim H, Elnashar M, Khidir A, Killawi A, Hammoud M, Al-Khal AL, \& Fetters MD (2017). Patient perspectives on language discordance during healthcare visits: Findings from the extremely high-density multicultural state of Qatar. Journal of Health Communication, 22(4), 355-363. 10.1080/10810730.2017.1296507 [PubMed: 28339340]

Al-Amer R, Ramjan L, Glew P, Darwish M, \& Salamonson Y (2016). Language translation challenges with Arabic speakers participating in qualitative research studies. International Journal of Nursing Studies, 54, 150-157. 10.1016/j.ijnurstu.2015.04.010 [PubMed: 25936733]

Allan HT, \& Westwood S (2016). English language skills requirements for internationally educated nurses working in the care industry: Barriers to UK registration or institutionalised discrimination? International Journal of Nursing Studies, 54, 1-4. 10.1016/j.ijnurstu.2014.12.006 [PubMed: 25597644]

Aroian KJ, Wu B, \& Tran TV (2005). Health care and social service use among Chinese immigrant elders. Research in Nursing \& Health, 28(2), 95-105. 10.1002/nur.20069 [PubMed: 15779058] 
Benitez I, \& Padilla J-L (2014). Analysis of nonequivalent assessments across different linguistic groups using a mixed methods approach: Understanding the causes of differential item functioning by cognitive interviewing. Journal of Mixed Methods Research, 8(1), 52-68.

$10.1177 / 1558689813488245$

Benitez I, Padilla JL, van de Vijver, \& Cuevas A (2018). What cognitive interviews tell us about bias in cross-cultural research. Field Methods, 30(4), 277-294. 10.1177/1525822X18783961

Bloemraad I, \& Sheares A (2017). Understanding membership in a world of global migration: (How) does citizenship matter? International Migration Review, 51(4), 823-867. 10.1111/imre.12354

Brzostek T, Brzyski P, Kozka M, Squires A, Przewozniak L, Cisek M, ... Ogarek M (2015). Research lessons from implementing a national nursing workforce study. International Nursing Review, 62(3), 412-420. 10.1111/inr.12191 [PubMed: 25918981]

Brzyski P, Kozka M, Squires A, \& Brzostek T (2016). How factor analysis results may change due to country context. Journal of Nursing Scholarship, 48(6), 598-607. 10.1111/jnu.12249 [PubMed: 27706893]

Cha E-S, Kim KH, \& Erlen JA (2007). Translation of scales in cross-cultural research: Issues and techniques. Journal of Advanced Nursing, 58(4), 386-395. 10.1111/j.1365-2648.2007.04242.x [PubMed: 17442038]

Chapple, \& Ziebland S (2018). Methodological and practical issues in cross-national qualitative research: Lessons from the literature and a comparative study of the experiences of people receiving a diagnosis of cancer. Qualitative Health Research, 28(5), 789-799. 10.1177/1049732317736284 [PubMed: 29094645]

Chidarikire S, Cross M, Skinner I, \& Cleary M (2018). Navigating nuances of language and meaning: Challenges of cross-language ethnography involving shona speakers living with schizophrenia. Qualitative Health Research, 28(6), 927-938. 10.1177/1049732318758645 [PubMed: 29468930]

Choi BK, Bjorner JB, Ostergren P-O, Clays E, Houtman I, Punnett L, ... Karasek R (2009). Crosslanguage differential item functioning of the job content questionnaire among European countries: The JACE study. International Journal of Behavioral Medicine, 16(2), 136-147. 10.1007/ s12529-009-9048-2 [PubMed: 19575297]

Clark L, Birkhead AS, Fernandez C, \& Egger MJ (2017). A transcription and translation protocol for sensitive cross-cultural team research. Qualitative Health Research, 27(12), 1751-1764. 10.1177/1049732317726761 [PubMed: 28936930]

Cohen B, Vawdrey DK, Liu J, Caplan D, Furuya EY, Mis FW, \& Larson E (2015). Challenges associated with using large data sets for quality assessment and research in clinical settings. Policy, Politics \& Nursing Practice, 16(3-4), 117-124. 10.1177/1527154415603358

Court D, \& Abbas R (2013). Whose interview is it, anyway? Methodological and ethical challenges of insider-outsider research, multiple languages and dual-researcher cooperation. Qualitative Inquiry, 19(6), 480-488. 10.1177/1077800413482102

Croot EJ, Lees J, Grant G, Barbour RS, Bradby H, Croot EJ, ... Poon M-K-L (2011). Evaluating standards in cross-language research: A critique of Squires' criteria. International Journal of Nursing Studies, 48(8), 1002-1011. 10.1016/j.ijnurstu.2011.04.007 [PubMed: 21571272]

Czaika M, \& de Haas H (2013). The globalisation of migration. Has the world really become more migratory? Working Papers 68, International Migration Institute, Oxford University, Oxford.

Danesi M (1996). Teen talk: What are the implications for second-language teaching? Mosaic, 3(4), 110.

De Gagne, J. C, Oh J, So A, \& Kim S-S (2014). The healthcare experiences of Koreans living in North Carolina: A mixed methods study. Health \& Social Care in the Community, 22(4), 417-428. 10.1111/hsc. 12098 [PubMed: 24621370]

Dowsey MM, Broadhead ML, Stoney JD, \& Choong PF (2009). Outcomes of total knee arthroplasty in English- versus non-English-speaking patients. Journal of Orthopaedic Surgery, 17(3), 305-309. 10.1177/230949900901700312 [PubMed: 20065369]

Durstenfeld MS, Ogedegbe O, Katz SD, Park H, \& Blecker S (2016). Racial and ethnic differences in heart failure readmissions and mortality in a large municipal healthcare system. JACC: Heart Failure, 4(11), 885-893. 10.1016/jjchf.2016.05.008 [PubMed: 27395346] 
Erkut S (2010). Developing multiple language versions of instruments for intercultural research. Child Development Perspectives, 4(1), 19-24. 10.1111/j.1750-8606.2009.00111.x [PubMed: 21423824]

Esposito N (2001). From meaning to meaning: The influence of translation techniques on non-English focus group research. Qualitative Health Research, 11(4), 568-579. 10.1177/104973201129119217 [PubMed: 11521612]

Flaherty JA, Gaviria FM, Pathak D, Mitchell T, Wintrob R, Richman JA, \& Birz S (1988). Developing instruments for cross-cultural psychiatric research. The Journal of Nervous and Mental Disease, 176(5), 257-263. 10.1097/00005053-198805000-00001 [PubMed: 3367140]

Hull M (2016). Medical language proficiency: A discussion of interprofessional language competencies and potential for patient risk. International Journal of Nursing Studies, 54, 158-172. 10.1016/j.ijnurstu.2015.02.015 [PubMed: 25863658]

Im E-O, Kim S, Tsai H-M, Nishigaki M, Yeo SA, Chee W, ... Mao JJ (2016). Practical issues in multilingual research. International Journal of Nursing Studies, 54, 141-149. 10.1016/j.ijnurstu. 2015.02.008 [PubMed: 25739658]

Im E-O, Lee SJ, Hu Y, Cheng C-Y, Iikura A, Inohara A, ... Chee W (2017). The use of multiple languages in a technology-based intervention study: A discussion paper. Applied Nursing Research, 38, 147-152. 10.1016/j.apnr.2017.10.011 [PubMed: 29241508]

International Organization for Migration (2017). World migration report 2018. Geneva: International Organization for Migration.

Johnson TP (2006). Methods and frameworks for crosscultural measurement. Medical Care, 44(11 Suppl 3), S17-S20. 10.1097/01.mlr.0000245424.16482.f1 [PubMed: 17060823]

Jones EG, \& Boyle JS (2011). Working with translators and interpreters in research: Lessons learned. Journal of Transcultural Nursing, 22(2), 109-115. 10.1177/1043659610395767 [PubMed: 21311084]

Kanchanachitra C, Lindelow M, Johnston T, Hanvoravongchai P, Lorenzo FM, Huong NL, ... dela Rosa JF (2011). Human resources for health in southeast Asia: Shortages, distributional challenges and international trade in health services. Lancet, 377(9767), 769-781. 10.1016/ S0140-6736(10)62035-1 [PubMed: 21269674]

Karliner LS, Kim SE, Meltzer DO, \& Auerbach AD (2010). Influence of language barriers on outcomes of hospital care for general medicine inpatients. Journal of Hospital Medicine, 5(5), 276-282. 10.1002/jhm.658 [PubMed: 20533573]

Larkin PJ, de Casterlé Dierckx, B., \& Schotsmans P (2007). Multilingual translation issues in qualitative research: Reflections on a metaphorical process. Qualitative Health Research, 17(4), 468-476. 10.1177/1049732307299258 [PubMed: 17416700]

Levin ME (2006). Language as a barrier to care for Xhosa-speaking patients at a South African paediatric teaching hospital. South African Medical Journal, 96(10), 1076-1079 http:// www.ncbi.nlm.nih.gov/pubmed/17164939. [PubMed: 17164939]

Lincoln YS, González y González, E. M, \& Aroztegui Massera C (2016). "Spanish Is a Loving Tongue ... ": Performing Qualitative Research Across Languages and Cultures. Qualitative Inquiry, 22(7), 531-540. 10.1177/1077800416636148

Liu K, Squires A, \& You, L.-M. (2011). A pilot study of a systematic method for translating patient satisfaction questionnaires. Journal of Advanced Nursing, 67(5), 1012-1021. 10.1111/j. 1365-2648.2010.05569.x [PubMed: 21261694]

Lynch KD (2007). Modeling role enactment: linking role theory and social cognition. Journal for the Theory of Social Behaviour, 37(4), 379-399. 10.1111/j.1468-5914.2007.00349.x

MacKenzie CA (2015). Filtered meaning: Appreciating linguistic skill, social position and subjectivity of interpreters in cross-language research. Qualitative Research, 16(2), 167-182. $10.1177 / 1468794115569564$

Mallinckrodt B, \& Wang C-C (2004). Quantitative methods for verifying semantic equivalence of translated research instruments: A Chinese version of the experiences in close relationships scale. Journal of Counseling Psychology, 51(3), 368-379. 10.1037/0022-0167.5L3.368

Maneesriwongul W, \& Dixon JK (2004). Instrument translation process: A methods review. Journal of Advanced Nursing, 48(2), 175-186. 10.1111/j.1365-2648.2004.03185.x [PubMed: 15369498] 
Montrul S (2010). Dominant language transfer in adult second language learners and heritage speakers. Second Language Research, 26(3), 293-327. 10.1177/0267658310365768

Morgeson FP, Delaney-Klinger K, \& Hemingway MA (2005). The importance of job autonomy, cognitive ability and job- related skill for predicting role breadth and job performance. The Journal of Applied Psychology, 90(2), 399-406. 10.1037/0021-9010.90.2.399 [PubMed: 15769248]

Müller A (2016). Language proficiency and nursing registration. International Journal of Nursing Studies, 54, 132-140. 10.1016/j.ijnurstu.2015.01.007 [PubMed: 25704372]

National Academies of Sciences Engineering and Medicine (2017). Accounting for social risk factors in medicare payment. Kwan LY, Stratton K, \& Steinwachs DM, (Eds.). Washington, DC: National Academies Press 10.17226/23635

O'Halloran KL, Tan S, Pham D-S, Bateman J, \& Vande Moere A (2018). A digital mixed methods research design: Integrating multimodal analysis with data mining and information visualization for big data analytics. Journal of Mixed Methods Research, 12(1), 11-30. $10.1177 / 1558689816651015$

Park H, Sha MM, \& Willis G (2016). Influence of english-language proficiency on the cognitive processing of survey questions. Field Methods, 28(4), 415-430. 10.1177/1525822X16630262

Paulus TM, Jackson K, \& Davidson J (2017). Digital Tools for Qualitative Research: Disruptions and Entanglements. Qualitative Inquiry, 23(10), 751-756. 10.1177/1077800417731080

Perneger TV, Leplege A, \& Etter JF (1999). Cross-cultural adaptation of a psychometric instrument: Two methods compared. Journal of Clinical Epidemiology, 52(11), 1037-1046. [PubMed: 10526997]

Poland BD (1995). Transcription quality as an aspect of rigor in qualitative research. Qualitative Inquiry, 1(3), 290-310. 10.1177/107780049500100302

Reeve BB, Willis G, Shariff-Marco SN, Breen N, Williams DR, Gee GC, ... Levin KY (2011). Comparing cognitive interviewing and psychometric methods to evaluate a racial/ethnic discrimination scale. Field Methods, 23(4), 397-419. https://doi.org/10.n77/1525822Xn416564 [PubMed: 29887759]

Reitig V, \& Squires A (2015). Building skills in North and Central America: Barriers and policy options toward harmonizing qualifications in nursing. Washington, DC: Migration Policy Institute.

Santos HPO, Black AM, \& Sandelowski M (2014). Timing of translation in cross-language qualitative research. Qualitative Health Research, 25(1), 134-144. 10.1177/1049732314549603 [PubMed: 25189538]

Savignon S (1997). Communicative competence: Theory and classroom practice (2nd ed.). New York, NY: McGraw-Hill.

Schwei RJ, Del Pozo S, Agger-Gupta N, Alvarado-Little W, Bagchi A, Chen AH, ... Jacobs EA (2016). Changes in research on language barriers in health care since 2003: A cross-sectional review study. International Journal of Nursing Studies, 54, 36-44. 10.1016/j.ijnurstu.2015.03.001 [PubMed: 25816944]

Shordike A, Hocking C, Pierce D, Wright-St. Clair V, Vittayakorn S, Rattakorn P, \& Bunrayong W (2010). Respecting regional culture in an international multi-site study: A derived etic method. Qualitative Research, 10(3), 333-355. 10.1177/1468794109360145

Shpilko I (2006). Russian-American health care: Bridging the communication gap between physicians and patients. Patient Education and Counseling, 64(1-3), 331-341. 10.1016/j.pec.2006.03.014 [PubMed: 16859861]

Sidani S, Guruge S, Miranda J, Ford-Gilboe M, \& Varcoe C (2010). Cultural adaptation and translation of measures: An integrated method. Research in Nursing \& Health, 33(2), 133-143. 10.1002/nur. 20364 [PubMed: 20069612]

Squires A (2008). Language barriers and qualitative nursing research: Methodological considerations. International Nursing Review, 55(3), 265-273. 10.1111/j.1466-7657.2008.00652.x [PubMed: 19522941]

Squires A (2009). Methodological challenges in cross-language qualitative research: A research review. International Journal of Nursing Studies, 46(2), 277-287. 10.1016/j.ijnurstu.2008.08.006 [PubMed: 18789799] 
Squires A, Aiken LH, van den Heede K, Sermeus W, Bruyneel L, Lindqvist R, ... Matthews A (2013). A systematic survey instrument translation process for multi-country, comparative health workforce studies. International Journal of Nursing Studies, 50(2), 264-273. 10.1016/j.ijnurstu. 2012.02.015 [PubMed: 22445444]

Squires A, Bruyneel L, Aiken LH, Van den Heede K, Brzostek T, Busse R, Sermeus W (2012). Crosscultural evaluation of the relevance of the HCAHPS survey in five European countries. International Journal for Quality in Health Care, 24(5), 470-475. 10.1093/intqhe/mzs040 [PubMed: 22807136]

Squires A, Finlayson C, Gerchow L, Cimiotti JP, Matthews A, Schwendimann R, ... Sermeus W (2014). Methodological considerations when translating "burnout". Burnout Research, 1(2), 5968. 10.1016/j.burn.2014.07.001 [PubMed: 25343131]

Stanley CA, \& Slattery P (2003). Who reveals what to whom? Critical reflections on conducting qualitative inquiry as an interdisciplinary, biracial, male/female research team. Qualitative Inquiry, 9(5), 705-728. 10.1177/1077800403253004

Temple B (2002). Crossed wires: Interpreters, translators and bilingual workers in cross-language research. Qualitative Health Research, 12(6), 844-854. [PubMed: 12109728]

Temple B (2005). Nice and tidy: Translation and representation. Sociological Research Online, 10(2), 1-10. 10.5153/sro. 1058

Temple B, \& Young A (2004). Qualitative research and translation dilemmas. Qualitative Research, 4(2), 161-178. 10.1177/1468794104044430

Tilley SA (2003). "Challenging” research practices: Turning a critical lens on the work of transcription. Qualitative Inquiry, 9(5), 750-773. 10.1177/1077800403255296

Tippu Z, Correa A, Liyanage H, Burleigh D, McGovern A, Van Vlymen J, ... De Lusignan S (2017). Ethnicity recording in primary care computerised medical record systems: An ontological approach. Journal of Innovation in Health Informatics, 23(4), 799 10.14236/jhi.v23i4.920

Weeks A, Swerissen H, \& Belfrage J (2007). Issues, challenges and solutions in translating study instruments. Evaluation Review, 31(2), 153-165. 10.1177/0193841X06294184 [PubMed: 17356181]

Whittal A, \& Lippke S (2016). Investigating patients with an immigration background in Canada: Relationships between individual immigrant attitudes, the doctor-patient relationship and health outcomes. BMC Public Health, 16(1), 23 10.1186/s12889-016-2695-8 [PubMed: 26753690]

Wong J-P-H, \& Poon M-K-L (2010). Bringing translation out of the shadows: Translation as an issue of methodological significance in cross-cultural qualitative research. Journal of Transcultural Nursing, 21(2), 151-158. https://doi.org/10.n77/1043659609357637 [PubMed: 20220035]

Xian H (2008). Lost in translation? Language, culture and the roles of translator in cross-cultural management research. Qualitative Research in Organizations and Management: An International Journal, 3(3), 231-245. 10.1108/17465640810920304

Yu DSF, Lee DTF, \& Woo J (2004). Issues and challenges of instrument translation. Western Journal of Nursing Research, 26(3), 307-320. 10.1177/0193945903260554 [PubMed: 15068554] 